\title{
A Note on the Almost Sure Central Limit Theorem for Partial Sums of $\rho^{-}$-Mixing Sequences
}

\author{
Feng Xu, Qunying Wu \\ College of Science, Guilin University of Technology, Guilin, China \\ Email: xufeng34@163.com, way666@glut.edu.cn
}

Received 27 July 2015; accepted 18 August 2015; published 21 August 2015

Copyright (C) 2015 by authors and Scientific Research Publishing Inc.

This work is licensed under the Creative Commons Attribution International License (CC BY).

http://creativecommons.org/licenses/by/4.0/

(c) () Open Access

\section{Abstract}

Let $\left\{X_{n}\right\}_{n \in N}$ be a strictly stationary sequence of $\boldsymbol{\rho}^{-}$-mixing random variables. We proved the almost sure central limit theorem, containing the general weight sequences, for the partial sums $S_{n} / \sigma_{n}$, where $S_{n}=\sum_{i=1}^{n} X_{i}, \sigma_{n}^{2}=E S_{n}^{2}$. The result generalizes and improves the previous results.

\section{Keywords}

\section{$\rho^{-}$-Mixing Sequences, Partial Sums, Almost Sure Central Limit Theorem}

\section{Introduction}

Let $C$ be a class of functions which are coordinatewise increasing. For a random variable $X$, define

$$
\|X\|_{p}=\left(\mathrm{E}|X|^{p}\right)^{1 / p} .
$$

For two nonempty disjoint sets $S, T \subset N$, we define $\operatorname{dist}(S, T)$ to be $\min \{|j-k| ; j \in S, k \in T\}$. Let $\sigma(S)$ be the $\sigma$-field generated by $\left\{X_{k}, k \in S\right\}$, and define $\sigma(T)$ similarly.

A sequence $\left\{X_{n}, n \geq 1\right\}$ is called negatively associated (NA) if for ever pair of disjoint subsets $S, T$ of $N$,

$$
\operatorname{cov}\left\{f\left(X_{i}, i \in S\right), g\left(X_{j}, j \in T\right)\right\} \leq 0,
$$

where $f, g \in \mathscr{C} .\left\{X_{n}, n \geq 1\right\}$ is called $\rho^{*}$-mixing, if

$$
\rho^{*}(k)=\sup \{\rho(S, T) ; S, T \subset N, \operatorname{dist}(S, T) \geq k\} \rightarrow 0, k \rightarrow \infty,
$$


where

$$
\rho(S, T)=\sup \left\{\frac{|\mathrm{E}(f-\mathrm{E} f)(g-\mathrm{E} g)|}{\|f-\mathrm{E} f\|_{2} \cdot\|g-\mathrm{E} g\|_{2}} ; f \in L_{2}(\sigma(S)), f \in L_{2}(\sigma(T))\right\} .
$$

Definition 1. [1] A sequence $\left\{X_{n}, n \geq 1\right\}$ is called $\rho^{-}$-mixing, if

$$
\rho^{-}(k)=\sup \left\{\rho^{-}(S, T): \operatorname{dist}(S, T) \geq k, S, T \subset N\right\} \rightarrow 0 \text {, as } k \rightarrow \infty,
$$

where

$$
\rho^{-}(S, T)=0 \vee \sup \left\{\frac{\operatorname{cov}\left\{f\left(X_{i}, i \in S\right), g\left(X_{j}, t \in T\right)\right\}}{\sqrt{\operatorname{var}\left\{f\left(X_{i}, i \in S\right)\right\} \operatorname{var}\left\{g\left(X_{j}, t \in T\right)\right\}}} ; f, g \in \mathcal{C}\right\} .
$$

The definition of NA is given by Joag-Dev and Proschan [2], and the concept of $\rho^{*}$-mixing random variables is given by Kolmogorov and Rozanov [3]. In 1999, the concept of $\rho^{-}$-mixing random variables was introduced initially by Zhang and Wang [1]. Obviously, $\rho^{-}$-mixing random variables include NA and $\rho^{*}$-mixing random variables, which have a lot of applications. Their limit properties have received more and more attention recently, and a number of results have been obtained, such as Zhang and Wang [1] for Rosenthal-type moment inequality and Marcinkiewicz-Zygmund law of large numbers, Zhang [4] for the central limit theorems of random fields, Wang and $\mathrm{Lu}[5]$ for the weak convergence theorems.

Starting with Brosamler [6] and Schatte [7], in the last two decades several authors investigated the almost sure central limit theorem (ASCLT) for partial sums $S_{n} / \sigma_{n}$ of random variables. We refer the reader to Brosamler [6], Schatte [7], Lacey and Philipp [8], Ibragimov and Lifshits [9], Berkes and Csáki [10], Hörmann [11] and $\mathrm{Wu}$ [12]. The simplest form of the ASCLT [6]-[8] reads as follows: let $\left\{X_{n} ; n \geq 1\right\}$ be i.i.d. random variables with mean 0 , variance $\sigma^{2}>0$ and partial sums $S_{n}$. Then

$$
\lim _{n \rightarrow \infty} \frac{1}{\log n} \sum_{k=1}^{n} \frac{1}{k} I\left(\frac{S_{k}}{\sigma \sqrt{k}} \leq x\right)=\Phi(x) \text { a.s. for any } x \in R .
$$

where $I$ denotes indicator function, and $\Phi(x)$ is the standard normal distribution function. For other version of $\rho^{-}$-mixing sequences, see [13]-[15].

The purpose of this article is to study and establish the ASCLT, containing the general weight sequences, for partial sums of $\rho^{-}$-mixing sequence. Our results not only generalize and improve those on ASCLT previously obtained by Brosamler [6], Schatte [7] and Lacey and Philipp [8] from the i.i.d. case to $\rho^{-}$-mixing sequences, but also expand the scope of the weights from $1 / k$ to $\exp \left(\log ^{\alpha} k\right) / k, 0 \leq \alpha<1 / 2$.

Throughout this paper, $a_{n} \sim b_{n}$ means $\lim _{n \rightarrow \infty} a_{n} / b_{n}=1$; and set the positive absolute constant $c$ to vary from line to line.

Theorem 1. Let $\left\{X_{n}\right\}_{n \in N}$ be a strictly stationary $\rho^{-}$-mixing sequence with $\mathrm{E} X_{1}=0,0<\mathrm{E}\left|X_{1}\right|^{r}<\infty$ for a certain $r>2$, and denote $S_{n}=\sum_{i=1}^{n} X_{i}, \sigma_{n}^{2}=\mathrm{ES}_{n}^{2}$. Assume that

(a) $\sigma^{2}=\mathrm{E} X_{1}^{2}+2 \sum_{k=2}^{\infty} \operatorname{cov}\left(X_{1}, X_{k}\right)>0$,

(b) $\sum_{k=2}^{\infty}\left|\operatorname{cov}\left(X_{1}, X_{k}\right)\right|<\infty$,

(c) $\sum_{k=1}^{\infty} \frac{\rho^{-}(k)}{k}<\infty$.

Suppose $0 \leq \alpha<1 / 2$ and set

$$
d_{k}=\frac{\exp \left(\log ^{\alpha} k\right)}{k}, D_{n}=\sum_{k=1}^{n} d_{k} \text {. }
$$

then 


$$
\lim _{n \rightarrow \infty} \frac{1}{D_{n}} \sum_{k=1}^{n} d_{k} I\left(\frac{S_{k}}{\sigma_{n}} \leq x\right)=\Phi(x) \text { a.s. for any } x \in \mathrm{R} .
$$

Remark 1. By the terminology of summation procedures (cf. [16], p. 35), Theorem 1 remains valid if we replace the weight sequence $\left\{d_{k}\right\}_{k \geq 1}$ by any $\left\{d_{k}^{*}\right\}_{k>1}$ such that $0 \leq d_{k}^{*} \leq d_{k}$ and $\sum_{k \geq 1} d_{k}^{*}=\infty$.

Remark 2. $\rho^{-}$-mixing random variables include NA and $\rho^{*}$-mixing random variables, so for NA and $\rho^{*}$-mixing random variables sequences Theorem 1 also holds.

Remark 3. Essentially, the open problem that whether Theorem 1 holds for $1 / 2 \leq \alpha<1$ still remains open.

\section{Some Lemmas}

Lemma 1. [4] Let $\left\{X_{n}, n \geq 1\right\}$ be a weakly stationary $\rho^{-}$-mixing sequence with $\mathrm{E} X_{n}=0,0<\mathrm{E} X_{1}^{2}<\infty$, and $\sigma^{2}=\mathrm{E} X_{1}^{2}+2 \sum_{k=2}^{\infty} \operatorname{cov}\left(X_{1}, X_{k}\right)>0, \sum_{k=2}^{\infty}\left|\operatorname{cov}\left(X_{1}, X_{k}\right)\right|<\infty$, then

$$
\frac{\sigma_{n}^{2}}{n} \rightarrow \sigma^{2}, \quad \frac{S_{n}}{\sigma_{n}} \stackrel{d}{\longrightarrow} \mathcal{N} \text {, as } n \rightarrow \infty,
$$

where $\mathcal{N}$ denotes the standard normal random variable.

Lemma 2. [5] For a positive real number $q \geq 2$, if $\left\{X_{n}, n \geq 1\right\}$ is a sequence of $\rho^{-}$-mixing random variables with $\mathrm{E} X_{i}=0, \mathrm{E}\left|X_{i}\right|^{q}<\infty$ for every $i \geq 1$, then for all $n \geq 1$, there is a positive constant $c=c\left(q, \rho^{-}(\cdot)\right)$ such that

$$
\mathrm{E}\left(\max _{1 \leq j \leq n}\left|S_{j}\right|^{q}\right) \leq c\left(\sum_{i=1}^{n} \mathrm{E}\left|X_{i}\right|^{q}+\left(\sum_{i=1}^{n} \mathrm{E} X_{i}^{2}\right)^{q / 2}\right) .
$$

Lemma 3. [17] Let $\left\{X_{n}, n \geq 1\right\}$ be a weakly stationary $\rho^{-}$-mixing sequence. Assume $\sup \mathrm{E}\left|X_{n}\right|^{r}<\infty$. Then for any bounded Lipschitz function $f: R \rightarrow R$, We have

$$
|\operatorname{cov}| f\left|\frac{S_{i}}{\sigma_{i}}\right|, f\left|\frac{S_{j}}{\sigma_{j}}\right||| \leq c\left|-\frac{1}{\sigma_{i} \sigma_{j}} \sum_{l=1}^{i} \sum_{m=2 i+1}^{2 i+l} \operatorname{cov}\left(X_{l}, X_{m}\right)+8 \rho^{-}(i)+2 \frac{\sigma_{2 i}}{\sigma_{j}}\right|
$$

Lemma 4. Let $\left\{\xi, \xi_{n}\right\}_{n \in N}$ be a sequence of uniformly bounded random variables. Assume that $\sum_{k=1}^{\infty} \frac{\rho^{-}(k)}{k}<\infty$, and existing constants $c>0$ and $\varepsilon>0$ such that

$$
\left|\mathrm{E} \xi_{k} \xi_{l}\right| \leq c\left(\rho^{-}(k)+\left(\frac{k}{l}\right)^{\varepsilon}\right) \text {, for } 1 \leq 2 k<l,
$$

then

$$
\lim _{n \rightarrow \infty} \frac{1}{D_{n}} \sum_{k=1}^{n} d_{k} \xi_{k}=0 \text { a.s., }
$$

where $d_{k}$ and $D_{n}$ are defined by (2).

Proof. Set $T_{n}=\frac{1}{D_{n}} \sum_{k=1}^{n} d_{k} \xi_{k}$, we get

$$
\begin{aligned}
\mathrm{ET}_{n}^{2} & =\frac{1}{D_{n}^{2}} \mathrm{E}\left(\sum_{k=1}^{n} d_{k} \xi_{k}\right)^{2} \leq \frac{1}{D_{n}^{2}} \sum_{1 \leq k \leq l \leq n, 2 k \geq l} d_{k} d_{l}\left|\mathrm{E} \xi_{k} \xi_{l}\right|+\frac{1}{D_{n}^{2}} \sum_{1 \leq k \leq l \leq n, 2 k<l} d_{k} d_{l}\left|\mathrm{E} \xi_{k} \xi_{l}\right| \\
& :=\frac{1}{D_{n}^{2}} T_{n 1}+\frac{1}{D_{n}^{2}} T_{n 2} .
\end{aligned}
$$

Firstly we estimate $T_{n 1}$. Since $\xi_{k}$ is a bounded random variable, we get 


$$
T_{n 1} \leq c \sum_{k=1}^{n} \sum_{l=k}^{2 k} d_{k} d_{l} \leq c \exp \left(\log ^{\alpha} n\right) \sum_{k=1}^{n} d_{k} \sum_{l=k}^{2 k} \frac{1}{l} \leq c D_{n} \exp \left(\log ^{2} n\right)
$$

Now we estimate $T_{n 2}$. By the conditions $\left|\mathrm{E} \xi_{k} \xi_{l}\right| \leq c\left(\rho^{-}(k)+\left(\frac{k}{l}\right)^{\varepsilon}\right)$ for $l>2 k$, we get

$$
\begin{aligned}
T_{n 2} & =\sum_{1 \leq k \leq l \leq n, 2 k<l} d_{k} d_{l}\left|\mathrm{E} \xi_{k} \xi_{l}\right| \leq c \sum_{l=2}^{n} \sum_{k=1}^{l-1} d_{k} d_{l} \rho^{-}(k)+c \sum_{l=2}^{n} \sum_{k=1}^{l-1} d_{k} d_{l}\left(\frac{k}{l}\right)^{\varepsilon} \\
& \leq c \sum_{l=1}^{n} d_{l} \sum_{k=1}^{n} d_{k} \rho^{-}(k)+c \sum_{l=1}^{n} \sum_{k=1}^{l} d_{k} d_{l}\left(\frac{k}{l}\right)^{\varepsilon}:=A_{1}+A_{2} .
\end{aligned}
$$

By condition $\sum_{k=1}^{\infty} \frac{\rho^{-}(k)}{k}<\infty$, we obtain

$$
A_{1} \leq c \exp \left(\log ^{\alpha} n\right) \sum_{l=1}^{n} d_{l} \sum_{k=1}^{n} \frac{\rho^{-}(k)}{k} \leq c D_{n} \exp \left(\log ^{\alpha} n\right)
$$

and

$$
A_{2} \leq c \sum_{l=2}^{n} \sum_{k=1}^{l} \frac{\exp \left(\log ^{\alpha} l\right)}{l^{1+\varepsilon}} \cdot \frac{\exp \left(\log ^{\alpha} k\right)}{k^{1-\varepsilon}} \leq c \exp \left(\log ^{\alpha} n\right) \sum_{l=1}^{n} \frac{\exp \left(\log ^{\alpha} l\right)}{l^{1+\varepsilon}} \cdot \frac{l^{\varepsilon}}{\varepsilon} \leq c D_{n} \exp \left(\log ^{\alpha} n\right) .
$$

Since $D_{n} \sim \frac{1}{\alpha} \log ^{1-\alpha} n \exp \left(\log ^{\alpha} n\right)$ and $\log D_{n} \sim \log ^{\alpha} n$ for $0<\alpha<1 / 2$ from the proof of Lemma 2.2 in $\mathrm{Wu}$ [18], we have, as $n \rightarrow \infty$,

$$
\exp \left(\log ^{\alpha} n\right) \sim \frac{\alpha D_{n}}{\left(\log D_{n}\right)^{(1-\alpha) / \alpha}} \sim \frac{\alpha D_{n}}{\log ^{1-\alpha} n}
$$

Thus

$$
\mathrm{ET}_{n}^{2} \leq \frac{1}{D_{n}^{2}}\left(T_{n 1}+A_{1}+A_{2}\right)=c \frac{\exp \left(\log ^{\alpha} n\right)}{D_{n}} \leq \frac{c}{\log ^{1-\alpha} n} .
$$

Let $n_{k}=\exp \left(k^{\tau}\right), \tau>1 /(1-\alpha)$, we get

$$
\sum_{K=1}^{\infty} P\left(\left|T_{n_{k}}\right|>\varepsilon\right) \leq c \sum_{k=1}^{\infty} \mathrm{E} T_{n_{k}}^{2} \leq c \sum_{k=1}^{\infty} \frac{1}{k^{(1-\alpha) \tau}}<\infty .
$$

By Borel-Cantelli lemma,

$$
T_{n_{k}} \rightarrow 0 \text { a.s., } k \rightarrow \infty .
$$

For any $n$, existing $n_{k}$ and $n_{k+1}$ such that $n_{k}<n \leq n_{k+1}$, then, by $\left|\xi_{i}\right| \leq c$ for any $i$,

$$
\left|T_{n}\right| \leq\left|\frac{1}{D_{n_{k}}} \sum_{i=1}^{n_{k}} d_{i} \xi_{i}\right|+\frac{1}{D_{n_{k}}} \sum_{i=n_{k}+1}^{n_{k+1}} d_{i}\left|\xi_{i}\right| \leq\left|T_{n_{k}}\right|+c\left(\frac{D_{n_{k+1}}-D_{n_{k}}}{D_{n_{k}}}\right) \rightarrow 0 \text { a.s. } n \rightarrow \infty,
$$

from $\frac{D_{n_{k+1}}}{D_{n_{k}}} \sim \frac{\exp \left((k+1)^{\tau}\right)}{\exp \left(k^{\tau}\right)} \sim \exp \left((k+1)^{\tau}\left(1-\left(\frac{k}{k+1}\right)^{\tau}\right)\right) \rightarrow 1$. i.e., (4) holds. This completes the proof of Lemma 4. 


\section{Proof}

Proof of Theorem 1. By Lemma 1, we have

$$
\frac{S_{k}}{\sigma_{k}} \stackrel{d}{\longrightarrow} \mathcal{N} \text {, as } k \rightarrow \infty \text {. }
$$

This implies that for any $g(x)$ which is a bounded function with bounded continuous derivatives,

$$
\mathrm{E} g\left(\frac{S_{k}}{\sigma_{k}}\right) \rightarrow \operatorname{Eg}(\mathcal{N}), \text { as } k \rightarrow \infty,
$$

Hence, by the Toeplitz lemma, we obtain

$$
\lim _{n \rightarrow \infty} \frac{1}{D_{n}} \sum_{k=1}^{n} d_{k} \mathrm{Eg}\left(\frac{S_{k}}{\sigma_{k}}\right)=\mathrm{Eg}(\mathcal{N}) .
$$

In the other hand, from Theorem 7.1 of Billingsley [19] and Section 2 of Peligrad and Shao [20], we know that (3) is equivalent to

$$
\lim _{n \rightarrow \infty} \frac{1}{D_{n}} \sum_{k=1}^{n} d_{k} g\left(\frac{S_{k}}{\sigma_{k}}\right)=\operatorname{Eg}(\mathcal{N}) \text { a.s.. }
$$

Hence, to prove (3), it suffices to prove

$$
\lim _{n \rightarrow \infty} \frac{1}{D_{n}} \sum_{k=1}^{n} d_{k}\left(g\left(\frac{S_{k}}{\sigma_{k}}\right)-\mathrm{E} g\left(\frac{S_{k}}{\sigma_{k}}\right)\right)=0 \text { a.s., }
$$

for any $g(x)$ which is a bounded function with bounded continuous derivatives.

Let $k \geq 1$, define

$$
\xi_{k}=g\left(\frac{S_{k}}{\sigma_{k}}\right)-\mathrm{Eg}\left(\frac{S_{k}}{\sigma_{k}}\right) \text {. }
$$

For any $1 \leq 2 k<l$, we get,

$$
\begin{aligned}
\left|\mathrm{E} \xi_{k} \xi_{l}\right| & =\left|\operatorname{cov}\left(g\left(\frac{S_{k}}{\sigma_{k}}\right), g\left(\frac{S_{l}}{\sigma_{l}}\right)\right)\right| \\
& =\left|\operatorname{cov}\left(g\left(\frac{S_{k}}{\sigma_{k}}\right), g\left(\frac{S_{l}}{\sigma_{l}}\right)-g\left(\frac{\sum_{i=2 k+1}^{l} X_{i}}{\sigma_{l}}\right)\right)\right|+\left|\operatorname{cov}\left(g\left(\frac{S_{k}}{\sigma_{k}}\right), g\left(\frac{\sum_{i=2 k+1}^{l} X_{i}}{\sigma_{l}}\right)\right)\right| \\
& :=I_{1}+I_{2} .
\end{aligned}
$$

Firstly we estimate $I_{1}$. By Lemma $1 \frac{\sigma_{n}^{2}}{n} \rightarrow \sigma^{2}$, we note that certain $n_{0} \in N, 0<\varepsilon<\sigma$ exist such that $\frac{1}{\sigma_{n}} \leq \frac{1}{(\sigma-\varepsilon) \sqrt{n}}$ as $n>n_{0}$. Since $g$ is a bounded Lipschitz function, i.e., there exists a constant $c>0$ such that $|g(x)| \leq c,|g(x)-g(y)| \leq c|x-y|$ for any $x, y \in \mathrm{R}$. By Jensen inequality, Lemma 2 and $\sigma<\infty$, we obtain that

$$
I_{1} \leq c \frac{\mathrm{E}\left|\sum_{i=1}^{2 k} X_{i}\right|}{\sqrt{l}} \leq c \frac{\sqrt{\mathrm{E}\left(\sum_{i=1}^{2 k} X_{i}\right)^{2}}}{\sqrt{l}} \leq c \frac{\sqrt{\left(\sum_{i=1}^{2 k} \mathrm{E} X_{i}^{2}\right)}}{\sqrt{l}} \leq c \frac{\sqrt{\left(\sum_{i=1}^{2 k} \mathrm{E} X_{1}^{2}\right)}}{\sqrt{l}} \leq c\left(\frac{k}{l}\right)^{1 / 2} .
$$

Now we estimate $I_{2}$. Note that $g$ is a bounded function with bounded continuous derivatives, so, by Lemma 
3, we have

$$
I_{2} \leq c \rho^{-}(k)
$$

So if $l>2 k$, combining with (6), (7), (8), we obtain

$$
\left|\mathrm{E} \xi_{k} \xi_{l}\right| \leq c\left(\left(\frac{k}{l}\right)^{1 / 2}+\rho^{-}(k)\right) .
$$

By Lemma 4, (5) holds.

This completes the proof of Theorem 1.1.

\section{Acknowledgments}

We thank the editor and the referee for their comments. This work is supported by National Natural Science Foundation of China (11361019).

\section{References}

[1] Zhang, L.X. and Wang, X.Y. (1999) Convergence Rates in the Strong Laws of Asymptotically Negatively Associated Random Fields. Applied Mathematics-A Journal of Chinese Universities Series B, 14, 406-416. http://dx.doi.org/10.1007/s11766-999-0070-6

[2] Joag-Dev, K. and Proschan, F. (1983) Negative Association of Random Variables with Applications. Annals of Statistics, 11, 286-295. http://dx.doi.org/10.1214/aos/1176346079

[3] Kolmogorov, A.N. and Rozanov, U.A. (1960) On Strong Mixing Conditions for Stationary Gaussian Processes. Theory of Probability and Its Applications, 5, 204-208. http://dx.doi.org/10.1137/1105018

[4] Zhang, L.X. (2000) Central Limit Theorems for Asymptotically Negatively Associated Random Fields. Acta Mathematica Sinica, 6, 691-710. http://dx.doi.org/10.1007/s101140000084

[5] Wang, J.F. and Lu, F.B. (2006) Inequalities of Maximum of Partial Sums and Weak Convergence for a Class of Weak Dependent Random Variables. Acta Mathematica Sinica, 22, 693-700. http://dx.doi.org/10.1007/s10114-005-0601-x

[6] Brosamler, G.A. (1988) An Almost Everywhere Central Limit Theorem. Mathematical Proceedings of the Cambridge Philosophical Society, 104, 561-574. http://dx.doi.org/10.1017/S0305004100065750

[7] Schatte, P. (1988) On Strong Versions of the Central Limit Theorem. Mathematische Nachrichten, 137, $249-256$. http://dx.doi.org/10.1002/mana.19881370117

[8] Lacey, M.T. and Philipp, W. (1990) A Note on the Almost Sure Central Limit Theorem. Statistics and Probability Letters, 9, 201-205. http://dx.doi.org/10.1016/0167-7152(90)90056-D

[9] Ibragimov, I.A. and Lifshits, M. (1998) On the Convergence of Generalized Moments in Almost Sure Central Limit Theorem. Statistics and Probability Letters, 40, 343-351. http://dx.doi.org/10.1016/S0167-7152(98)00134-5

[10] Berkes, I. and Csáki, E. (2001) A Universal Result in Almost Sure Central Limit Theory. Stochastic Processes and Their Applications, 94, 105-134. http://dx.doi.org/10.1016/S0304-4149(01)00078-3

[11] Hörmann, S. (2007) Critical Behavior in Almost Sure Central Limit Theory. Journal of Theoretical Probability, 20, 613-636. http://dx.doi.org/10.1007/s10959-007-0080-3

[12] Wu, Q.Y. (2011) Almost Sure Limit Theorems for Stable Distribution. Statistics and Probability Letters, 281, $662-672$. http://dx.doi.org/10.1016/j.spl.2011.02.003

[13] Zhang, M.D., Tan, X.L. and Zhang, Y. (2015) An Extension of Almost Sure Central Limit Theorem for Product of Partial Sums of $\rho^{-}$-Mixing Sequences. Journal of Beihua University (Natural Science), 16, 427-430.

[14] Tan, L.X., Zhang, Y. and Zhang, Y. (2012) An Almost Sure Central Limit Theorem of Products of Partial Sums for $\rho^{-}$-Mixing Sequences. Journal of Inequalities and Applications, 2012, 51-63.

[15] Zhou, G.Y. and Zhang, Y. (2014) Almost Sure Central Limit Theorem of Products of Sums of Partial Sums for $\rho^{-}$-Mixing Sequences. Journal of Jilin University (Science Edition), 50, 1129-1134.

[16] Chandrasekharan, K. and Minakshisundaram, S. (1952) Typical Means. Oxford University Press, Oxford.

[17] Zhou, H. (2005) A Note on the Almost Sure Central Limit Theorem for $\rho^{-}$-Mixing Sequences. Chinese Journal Zhejiang University (Science Edition), 32, C503-C505.

[18] Wu, Q.Y. (2012) A Note on the Almost Sure Limit Theorem for Self-Normalized Partial Sums of Random Variables in 
the Domain of Attraction of Thenormal Law. Journal of Inequalities and Applications, 2012, 17-26.

[19] Billingsley, P. (1968) Convergence of Probability Measures. Wiley, New York.

[20] Peligrad, M. and Shao, Q.M. (1995) A Note on the Almost Sure Central Limit Theorem for Weakly Dependent Random Variables. Statistics and Probability Letters, 22, 131-136. http://dx.doi.org/10.1016/0167-7152(94)00059-H 